\title{
Predição da porosidade drenável e disponibilidade de água para Cambissolos da Microrregião Campos das Vertentes, MG ${ }^{(1)}$
}

\author{
Carlos Rogério de Mello ${ }^{(2)}$, Geraldo César de Oliveira ${ }^{(2)}$, Daniel Furtado Ferreira ${ }^{(3)}$ \\ e José Maria de Lima(4) $^{(4)}$
}

\begin{abstract}
Resumo - O uso de equações para predição de algumas propriedades do solo é importante para redução do tempo e dos custos laboratoriais despendidos, especialmente para aplicação rápida e precisa desses valores em projetos agrícolas. A estimativa de uma propriedade do solo através de outras propriedades, que são obtidas de forma rotineira, deve ser contemplada. O objetivo deste trabalho foi obter equações que permitam estimar, com boa precisão, o grau de porosidade drenável e a disponibilidade total de água, a partir da análise textural, do teor de matéria orgânica e da densidade do solo, cuja determinação em laboratório é fácil e rápida. Para isto, trabalhou-se com Cambissolos da Microrregião Campos das Vertentes, MG, até a profundidade de $40 \mathrm{~cm}$, utilizando 36 pontos amostrais. Por meio do programa SAS, através do procedimento "Backward", foi possível ajustar equações que apresentaram boas predições das propriedades em estudo a partir de análises rotineiras de laboratório, o que servirá de suporte para futuros estudos na região analisada.
\end{abstract}

Termos para indexação: retenção de água, disponibilidade hídrica, propriedades hidráulicas do solo, modelos matemáticos.

\section{Prediction of drainable porosity and water-holding capacity for Inceptsol from Campos das Vertentes Region, Minas Gerais State, Brazil}

\begin{abstract}
The use of equations for predicting some soil properties is important for reducing time and laboratorial costs, especially for rapid and precise application of those values in agricultural projects. The estimation of one soil property through other properties which are obtained in a daily routine ought to be considered. The objective of this work was to obtain equations based on soil texture, soil organic matter content and bulk density that allow to estimate with good precision the grade of drainable porosity and the water-holding capacity. The methods for measuring these parameters are used in the routine of most laboratories of soil analisys. Soil texture, organic matter, and bulk density were measured in 36 samples from zero to $40 \mathrm{~cm}$ depth of an Inceptsol from the region of Campos das Vertentes, MG, Brazil. The results were compared to drainable porosity and water-holding capacity though the Backward procedure of SAS program. The final equations allowed a good estimate of both parameters, which can the useful for suport of future studies in the region analysed.
\end{abstract}

Index terms: water retention, water availability, soil hydraulic properties, mathematical models.

\section{Introdução}

O uso de modelos matemáticos para estimativa de propriedades do solo de determinação relativa-

\footnotetext{
(1) Aceito para publicação em 28 de novembro de 2001 .

(2) Universidade Federal de Lavras (Ufla), Dep. de Ciência do Solo, Caixa Postal 37, CEP 37200-000 Lavras, MG E-mail: crmello@ufla.br, gcesar@agro.ufg.br

(3) Ufla, Dep. de Ciências Exatas. Bolsista do CNPq. E-mail: danielff@ufla.br

(4) Ufla, Dep. de Ciência do Solo. Bolsista do CNPq. E-mail: jmlima@ufla.br
}

mente difícil com base em outras características, de fácil e rápida análise, tem sido freqüente em projetos hidroagrícolas, visando economia de tempo e redução de custos com análises laboratoriais (Sales et al., 1999).

Uma das propriedades físico-hídricas importantes para o manejo e estudo de fluxos hidráulicos no solo é a porosidade drenável, sendo vital na modelagem da contaminação do lençol freático e na drenagem propriamente dita. Esta propriedade é também conhecida como porosidade efetiva, e define-se como a fração da porosidade total na qual a água move-se livremente, equivalendo ao conteúdo de ar 
presente no solo na capacidade de campo (Beltran, 1986; Queiroz, 1995). Este parâmetro pode ser medido in situ monitorando-se alturas do lençol freático, e em projetos de drenagem já instalados, acompanhando-se descargas dos drenos em razão do tempo (Braun \& Kruijne, 1994). Em laboratório, há necessidade de saturação da amostra, colocação sob tensão correspondente à capacidade de campo, e em seguida, determinar as umidades correspondentes. Todas estas etapas podem levar mais de 72 horas (Embrapa, 1997). Alguns pesquisadores desenvolveram modelos matemáticos para predizer a porosidade drenável em função da condutividade hidráulica saturada, e não obtiveram êxito em tal método, haja vista que a condutividade hidráulica saturada possui alta variabilidade espacial. Porém, demonstraram que algumas propriedades, como textura, matéria orgânica e densidade aparente, podem explicar satisfatoriamente a porosidade drenável (Galdino, 1988; Libardi, 1995; Klein, 1998).

O conhecimento da disponibilidade total de água no solo é a parte principal de um projeto de irrigação, e demanda análises de retenção de água em laboratório, o que acarreta, da mesma forma, aumento de custos e tempo. O termo Disponibilidade Total de Água (DTA), de acordo com Reichardt (1988) e Klein (1998), refere-se à porção de água contida no solo entre a capacidade de campo e o ponto de murcha permanente, ou seja, a água retida pela matriz do solo, indispensável para o correto manejo da água em projetos de irrigação. A textura e matéria orgânica interferem diretamente na capacidade de retenção de água pelo solo. Saxton et al. (1986), Arruda et al. (1987), Centurion \& Andrioli (2000) e Fabian \& Ottoni Filho (2000) concluíram que estas propriedades correlacionam positivamente com a retenção (disponibilidade) de água de um solo, e relatam também, a importância da estrutura do solo neste contexto.

$\mathrm{O}$ fato de as hidrelétricas de Itutinga e Camargos estarem inseridas na Microrregião Campos das Vertentes, bem como de serem os Cambissolos muito representativos no entorno dos lagos (Brasil, 1983; Giarola et al., 1997), fazem com que este seja um trabalho relevante, dada a importância de um bom controle quantitativo e qualitativo da água nestes reservatórios, pois, estes solos têm sérios problemas ocasionados por escoamento superficial. Além disso, a presença de indústrias de cimento e siderurgia, depósitos de lixo e forte atividade agropastoril, já podem estar comprometendo a qualidade da água subsuperficial e, portanto, os resultados deste trabalho darão suporte a futuros estudos técnico-científicos para instalação de projetos de irrigação e drenagem e prevenção contra a contaminação ambiental.

O objetivo deste trabalho foi gerar equações matemáticas que permitam estimar, com boa precisão, a porosidade drenável e a disponibilidade total de água, a partir da análise textural, do teor de matéria orgânica e da densidade do solo.

\section{Material e Métodos}

Foram coletadas, aleatoriamente, amostras de 36 pontos $(0-20 ; 20-40 \mathrm{~cm})$ de três solos originados de diferentes materiais de origem: Filito, Micaxisto e Gnaisse - Granítico Leucocrático, classificados como Cambissolo Háplico Tb distrófico típico (Tabela 1). Mais informações a respeito dos solos em estudo podem ser encontradas em Oliveira (1993).

Desenvolveram-se curvas de retenção de amostras indeformadas, conforme Klute (1986), considerando como capacidade de campo a umidade do solo equivalente à tensão de $6 \mathrm{kPa}$ (Ferreira \& Marcos, 1983), e, como ponto de murcha permanente, a umidade na tensão de $15 \mathrm{kPa}$ (Klein, 1998). Desta forma, pode-se calcular a porosidade drenável, conforme Otto (1988) e Queiroz (1995):

$\mathrm{PD}=\theta_{\mathrm{S}}-\theta_{\mathrm{CC}}$

em que PD é a porosidade drenável $\left(\mathrm{cm} \mathrm{cm}^{-3}\right)$; $\theta_{\mathrm{S}}$ é a umidade, base volume, na saturação $\left(\mathrm{cm} \mathrm{cm}^{-3}\right) ; \theta_{\mathrm{CC}}$ é a umidade, base volume, na capacidade de campo $\left(\mathrm{cm} \mathrm{cm}^{-3}\right)$.

A disponibilidade total de água foi determinada segundo Klar (1988) e Bernardo (1989):

Tabela 1. Atributos químicos e físicos na profundidade de $0-20 \mathrm{~cm}$ e de $20-40 \mathrm{~cm}$ dos três Cambissolos em estu-

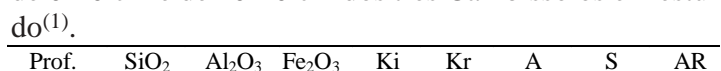

\begin{tabular}{rcccccccc} 
Prof. & $\mathrm{SiO}_{2}$ & $\mathrm{Al}_{2} \mathrm{O}_{3}$ & $\mathrm{Fe}_{2} \mathrm{O}_{3}$ & $\mathrm{Ki}$ & $\mathrm{Kr}$ & $\mathrm{A}$ & $\mathrm{S}$ & $\mathrm{AR}$ \\
$\mathrm{cm}$ & $--------~$ & $\left(\mathrm{~g} \mathrm{~kg}^{-1}\right)$ & ------- \\
\hline \multicolumn{7}{c}{ Cambissolo originado da alteração de Gnaisse-Granítico } & \\
$0-20$ & 110 & 190 & 40 & 1,0 & 0,5 & 330 & 160 & 510 \\
$20-40$ & 170 & 170 & 50 & 1,7 & 0,9 & 400 & 170 & 430 \\
\hline \multicolumn{7}{c}{ Cambissolo originado da alteração de Rochas Metapelíticas } \\
$0-20$ & 150 & 210 & 90 & 1,2 & 0,5 & 400 & 230 & 370 \\
$20-40$ & 180 & 240 & 100 & 1,2 & 0,6 & 400 & 230 & 370 \\
\hline \multicolumn{8}{c}{ Cambissolo originado da alteração de Filito } \\
$0-20$ & 240 & 260 & 130 & 1,5 & 1,2 & 680 & 160 & 160 \\
$20-40$ & 250 & 290 & 140 & 1,5 & 1,1 & 720 & 180 & 100 \\
\hline
\end{tabular}

(1)Oliveira (1993); Ki: relação molecular $\mathrm{SiO}_{2} / \mathrm{Al}_{2} \mathrm{O}_{3}$; Kr: relação molecular $\mathrm{SiO}_{2} /\left(\mathrm{Al}_{2} \mathrm{O}_{3}+\mathrm{Fe}_{2} \mathrm{O}_{3}\right)$; A: argila; S: silte; AR: areia. 


$$
\mathrm{DTA}=\theta_{\mathrm{CC}}-\theta_{\mathrm{PMP}}
$$

em que DTA é a disponibilidade total de água $\left(\mathrm{cm} \mathrm{cm}^{-3}\right)$; $\theta_{\text {PMP }}$ é a umidade, base volume, no ponto de murcha permanente $\left(\mathrm{cm} \mathrm{cm}^{-3}\right)$.

A análise textural foi feita pelo método de Boyoucos (Gee \& Bauder, 1986); a densidade do solo, pelo método do cilindro de Uhland (Embrapa, 1997); e carbono orgânico por meio de sua queima. A matéria orgânica foi obtida pela multiplicação do carbono orgânico por 1,724 (Walkley \& Black, 1934).

Na geração dos modelos, trabalhou-se com o Programa SAS (SAS Institute, 1985), usando o procedimento "Backward", o qual seleciona as variáveis que são significativas conforme o nível de probabilidade estipulado, ou seja, pode-se trabalhar com várias variáveis independentes, e o programa procura saber quais delas têm significância na estimativa da variável dependente em questão. As equações foram geradas por meio de regressão linear múltipla (mínimos quadrados) das variáveis selecionadas. $\mathrm{Na}$ avaliação da precisão das estimativas, consideraram-se o coeficiente de determinação ajustado ( $\mathrm{R}^{2}$ ajustado), o erro médio gerado pelos modelos, e a significância das variáveis (Prob > $|\mathrm{T}|$ ) a 5\% de probabilidade. Várias combinações das variáveis, bem como suas formas isoladas, foram introduzidas no modelo completo para obtenção de um modelo final, usando o referido procedimento estatístico. $\mathrm{O}$ erro foi calculado pela seguinte fórmula para cada ponto amostrado:

$\mathrm{e}=\frac{\left|\mathrm{V}_{\text {real }}-\mathrm{V}_{\text {predito }}\right|}{\mathrm{V}_{\text {real }}} \times 100$

em que e é o erro em cada ponto amostrado (\%); $\mathrm{V}_{\text {real }}$ é o valor real da variável aleatória em cada ponto amostrado; $\mathrm{V}_{\text {predito }}$ é valor predito pelos modelos no respectivo ponto amostrado.

Por meio da média aritmética destes valores, encontrase o erro médio gerado pelo modelo.

\section{Resultados e Discussão}

As propriedades do solo mais significativas na modelagem da porosidade drenável foram os porcentuais de argila e areia (PARG e PA, respectivamente) e a densidade do solo (DS); todas as variáveis criadas, a partir de combinações destas propriedades, foram significativas a 5\% de probabilidade (Tabela 2). Gupta \& Larson (1979), Galdino (1988) e Otto (1988) constataram comportamento semelhante, e verificaram que a matéria orgânica também pode ser significativa neste tipo de modelagem. Pela aná- lise matemática desenvolvida, não foi possível detectar influência da matéria orgânica no comportamento da porosidade drenável dos solos estudados, talvez por causa das pequenas variações observadas nos valores desta variável.

Os valores estimados ficaram próximos dos valores reais, podendo-se observar a pequena dispersão dos pontos em torno da reta 1:1 (Figura 1). Esta reta indica o que seria um ajuste perfeito, ou seja, quanto mais próximo dela, maior a precisão da estimativa (Wösten et al., 1990; Zhuang et al., 2001). O coeficiente de determinação ajustado foi de $88,02 \%$, com erro médio de $10,9 \%$, o que permite classificar a equação como boa estimadora da porosidade drenável (Gupta \& Larson, 1979; Sales et al., 1999).

A fim de predizer as umidades de saturação e capacidade de campo de horizontes superficiais, Abbaspour \& Moon (1992) ajustaram equações por meio de regressão múltipla e calcularam estas propriedades hídricas do solo em função da textura e densidade do solo, tais equações apresentaram ajustes satisfatórios com coeficientes de determinação de $55 \%$ e $70 \%$, respectivamente, para umidade de saturação e capacidade de campo. Desta forma, os resultados deste trabalho mostram que, aplicando-se as mesmas variáveis independentes (textura e densidade aparente), pode-se predizer a porosidade drenável de maneira mais precisa, utilizando-se apenas uma equação.

Quanto a disponibilidade total de água, houve alta significância apenas das variáveis relativas aos porcentuais de areia (PA), argila (PARG) e matéria orgânica (MO) (Tabela 3), conforme verificado por Saxton et al. (1986), Arruda et al. (1987) e Centurion

Tabela 2. Variáveis selecionadas pelo procedimento "Backward" com respectivas estimativas dos parâmetros e nível de significância a 5\% de probabilidade, da equação estimadora da porosidade drenável ${ }^{(1)}$.

\begin{tabular}{lcc}
\hline Variável & Estimativa dos parâmetros & Prob > $|\mathrm{t}|$ \\
\hline Intercepto & $-149,011$ & 0,0585 \\
Ln (PA) & 443,074 & 0,0135 \\
Ln (DS) & $-814,455$ & 0,0145 \\
Ln (PARG) xLn (PA) & $-129,868$ & 0,0172 \\
(PARG) & $-0,0569$ & 0,0033 \\
DSxPARG & 8,431 & 0,0097 \\
DSxPAxPARG & 0,1783 & 0,0215 \\
(PA) ${ }^{2}$ xPARG & $-0,00297$ & 0,0084 \\
\hline
\end{tabular}

(1)PA: porcentual de areia; DS: densidade do solo; PARG: porcentual de argila. 
\& Andrioli (2000), que realizaram estudos semelhantes com outros solos.

Os valores estimados ficaram próximos dos valores reais, mostrando uma dispersão ainda menor das

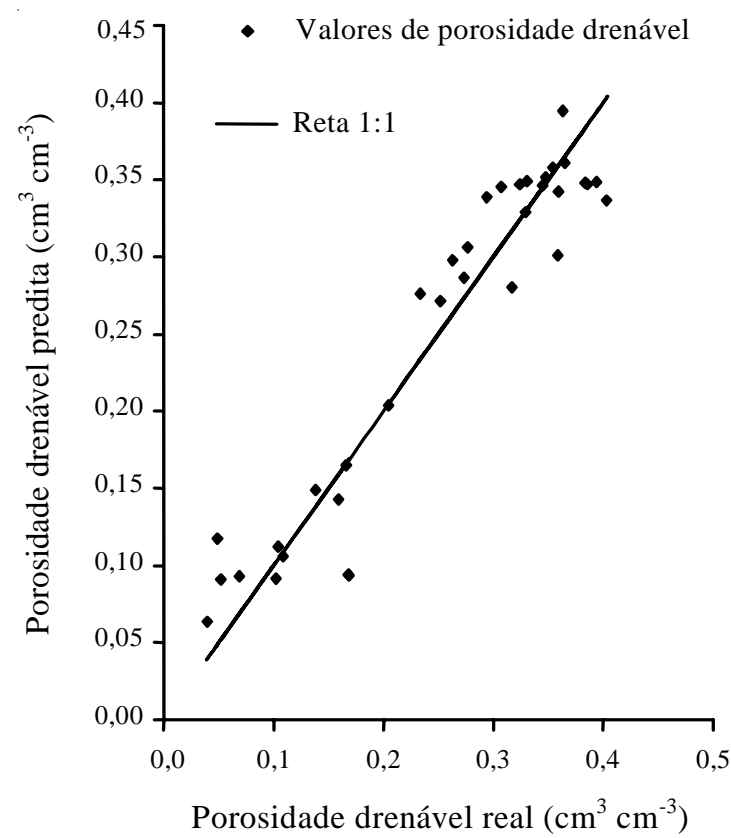

Figura 1. Representação dos valores de porosidade drenável preditos pela equação formada com as variáveis e os parâmetros encontrados na Tabela 2, em relação aos valores determinados em laboratório. $\mathrm{O}$ coeficiente de determinação, $\mathrm{R}^{2}$ ajustado, foi de $88,02 \%$ e o erro médio de $10,9 \%$.

Tabela 3. Variáveis com respectivas estimativas dos parâmetros e nível de significância a 5\% de probabilidade da equação preditora da disponibilidade total de água ajus$\operatorname{tada}^{(1)}$.

\begin{tabular}{lrc}
\hline Variável & Estimativa dos parâmetros & Prob $>|t|$ \\
\hline Intercepto & $-146.209,0$ & 0,0001 \\
PA & 722,241 & 0,0001 \\
PARG & $-1.073,397$ & 0,0001 \\
MO & $-379,298$ & 0,0002 \\
Ln (MO) & 472,859 & 0,0004 \\
$($ PA) & $-1,579$ & 0,0001 \\
$($ PARG) & 3,256 & 0,0001 \\
$($ PA)x(PARG) & $-30,104$ & 0,0001 \\
(PA) $^{2}$ x(PARG) & $-0,0845$ & 0,0001 \\
PAx(PARG) & 0,1339 & 0,0001 \\
$($ PA) & 2 x(PARG) & 0,0001 \\
Ln (PARG) & 0,00424 & 0,0001 \\
Ln (PA) & $47.179,0$ & 0,0001 \\
(MO) $^{2}$ & $4.875,726$ & 0,0001 \\
\hline
\end{tabular}

${ }^{(1)} \mathrm{PA}$ : porcentual de areia; PARG: porcentual de argila; MO: porcentual de matéria orgânica. estimativas em torno da reta 1:1 (Figura 2). O coeficiente de determinação ajustado foi de $92,4 \%$, e o erro médio foi de $6,9 \%$, permitindo, da mesma forma anterior, classificar a equação como boa preditora da disponibilidade total de água.

Saxton et al. (1986) geraram equações semelhantes para estimativa da disponibilidade total de água, ajustando de forma independente uma equação para a umidade, correspondente à capacidade de campo, e outra, relativa ao ponto de murcha permanente, ambas com alto coeficiente de determinação, em torno de $94 \%$, próximo ao obtido no presente trabalho. Esses autores trabalharam com maior quantidade de dados e consideraram somente os dados relativos à textura. Desta forma, comprova-se, por meio das análises matemáticas, que a matéria orgânica tem influência no potencial matricial do solo, e que é importante considerá-la no processo de modelagem da retenção total de água pela matriz do solo.

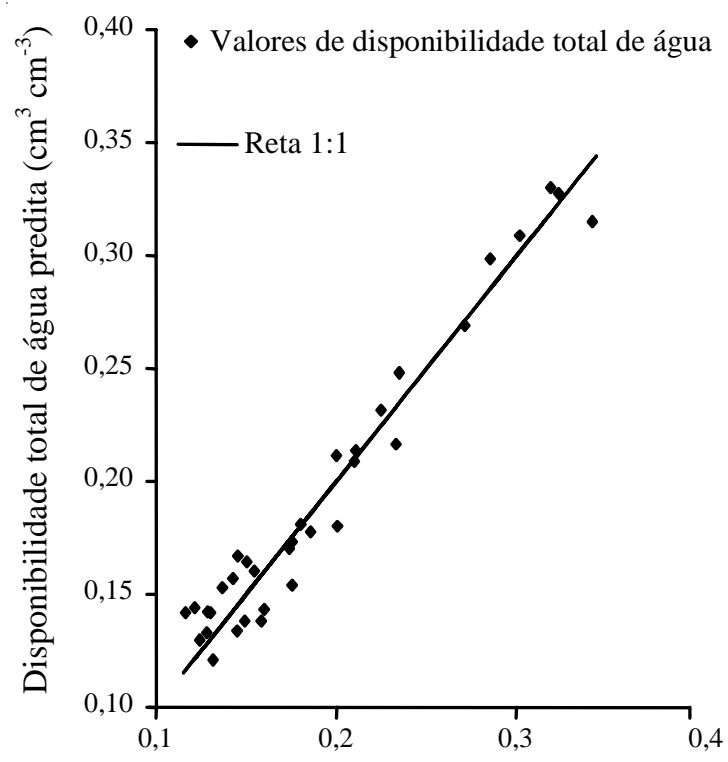

Disponibilidade total de água real $\left(\mathrm{cm}^{3} \mathrm{~cm}^{-3}\right)$

Figura 2. Representação dos valores de disponibilidade total de água preditos pela equação formada com as variáveis e os parâmetros encontrados na Tabela 3, em relação aos valores determinados em laboratório. O coeficiente de determinação, $\mathrm{R}^{2}$ ajustado, foi de $92,4 \%$ e o erro médio da predição foi de $6,9 \%$. 
A equação proposta para disponibilidade total de água é mais precisa com os valores preditos estando mais próximos da reta $1: 1$, proporcionando menores erros (Figuras 1 e 2). Isto reflete uma menor dificuldade no ajuste da equação proporcionada pela propriedade em estudo, ou seja, a DTA provavelmente possui menor variabilidade, em face dos efeitos aleatórios do processo, haja vista que a umidade correspondente à tensão de $15 \mathrm{kPa}$ não varia consideravelmente. O nível de significância de cada variável é bem menor comparativamente às variáveis estimadoras da porosidade drenável, e isto mostra a importância marcante da textura e matéria orgânica na DTA de um solo.

Quanto aos modelos propostos no presente trabalho, o uso do procedimento "Backward" possibilitou bons ajustes, tendo em vista os coeficientes de determinação e os erros proporcionados, o que demonstra precisão considerável, principalmente por se tratar de propriedades do solo que normalmente apresentam alta variabilidade. Estes resultados estão de acordo com os de Cornelis et al. (2001), que, ao analisarem a evolução de funções de pedotransferência para predição da retenção de umidade do solo, concluíram que o uso de modelagens estatísticas tem proporcionado grandes evoluções em termos de precisão, e são altamente válidos estudos desta natureza.

\section{Conclusões}

1. As equações propostas estimam com boa precisão a porosidade drenável e a disponibilidade total de água até a profundidade de $40 \mathrm{~cm}$ nos Cambissolos da região Campos das Vertentes, MG.

2. Essas equações permitem agilizar o conhecimento dessas duas propriedades físico-hídricas, importantes no estudo da dinâmica e manejo da água no solo.

\section{Referências}

ABBASPOUR, K. C.; MOON, D. E. Relationships between conventional field information and some soil properties measured in the laboratory. Geoderma, Amsterdam, v. 55, p. 119-140, 1992.
ARRUDA, F. B.; ZULLO JÚNIOR, J.; OLIVEIRA, J. B. Parâmetros de solo para o cálculo da água disponível com base na textura do solo. Revista Brasileira de Ciência do Solo, Campinas, v. 11, n. 1, p. 11-15, 1987.

BELTRAN, J. M. Drenaje agrícola. Madrid: Instituto Nacional de Reforma y Desarrollo Agrario, 1986. 239 p.

BERNARDO, S. Manual de irrigação. Viçosa, MG: UFV, 1989. 596 p.

BRASIL. Ministério de Minas e Energia. Departamento Nacional da Produção Mineral. Projeto RADAMBRASIL.

Folhas S. F. 23/24 Rio de Janeiro/Vitória. Rio de Janeiro: geologia, geomorfologia, pedologia, vegetação, uso potencial da terra. 1983. Rio de Janeiro, 1983. p. 780. (Levantamento de recursos naturais, 32).

BRAUN, H. M. H.; KRUIJNE, R. Soil conditions. In: RITZEMA, H. P. (Ed.). Drainage principles and applications. 2. ed. Wageningen: International Institute for Land Reclamation and Improvement, 1994. p. 77-110.

CENTURION, J. F.; ANDRIOLI, I. Regime hídrico de alguns solos de Jaboticabal. Revista Brasileira de Ciência do Solo, Viçosa, MG, v. 24, n. 4, p. 701-709, 2000.

CORNELIS, W. M.; RONSYN, J.; MEIRVENNE, M. van; HARTMANN, R. Evaluation of pedotransfer functions for predicting the soil moisture retention curve. Soil Science Society of America Journal, Madison, v. 65 , n. 3, p. 638-648, 2001.

EMBRAPA. Centro Nacional de Pesquisa de Solos (Rio de Janeiro, RJ). Manual de métodos de análise de solos. 2. ed. Rio de Janeiro, 1997. 212 p.

FABIAN, A. J.; OTTONI FILHO, T. B. Determinação de capacidade de campo in situ ou através de equações de regressão. Pesquisa Agropecuária Brasileira, Brasília, v. 35, n. 5, p. 1029-1036, maio 2000.

FERREIRA, M. M.; MARCOS, Z. Z. Estimativa da capacidade de campo de Latossolo Roxo distrófico e Regossolo através do ponto de inflexão da curva característica de umidade. Ciência e Prática, Lavras, v. 7, p. 96101, 1983.

GALDINO, S. Estimativa de macroporosidade em função de algumas características de solo de várzea. 1988. 61 f. Dissertação (Mestrado) - Universidade Federal de Viçosa, Viçosa.

Pesq. agropec. bras., Brasília, v. 37, n. 9, p. 1319-1324, set. 2002 
GEE, G. W.; BAUDER, J. W. Particle-size analysis. In: METHODS of soil analysis. Madison: American Society of Agronomy, 1986. p. 383-411.

GIAROLA, N. F. B.; CURI, N.; SIQUEIRA, J. O.; CHAGAS, C. S.; FERREIRA, M. M. Solos da região sob influência do reservatório da hidrelétrica de Itutinga/Camargos (MG): perspectiva ambiental. Belo Horizonte: Ufla/Cemig, 1997. 101 p.

GUPTA, S. C.; LARSON, W. E. Estimating soil water retention characteristics from particle size distribution, organic matter content, and bulk density. Water Resources Research, Washington, v. 20, p. 1633-1635, 1979.

KLAR, A. E. A água no sistema solo-planta-atmosfera. São Paulo: Nobel, 1988. 408 p.

KLEIN, V. A. Propriedades físico-hídrico-mecânicas de um Latossolo Roxo, sob diferentes sistemas de uso e manejo. 1998. 150 f. Tese (Doutorado) - Escola Superior de Agricultura Luiz de Queiroz, Piracicaba.

KLUTE, A. Water retention: laboratory methods. In: METHODS of soil analysis. Madison: American Society of Agronomy, 1986. p. 635-686.

LIBARDI, P. L. Dinâmica da água no solo. Piracicaba: [s.n.], 1995. 497 p.

OLIVEIRA, G. C. de. Cambissolos da microrregião Campos da Mantiqueira, MG: caracterização físicohídrica e interpretação para manejo. 1993. 62 f. Dissertação (Mestrado) - Universidade Federal de Lavras, Lavras.

OTTO, S. R. L. Estimativa da porosidade drenável em função de propriedades de um solo orgânico. 1988.
89 f. Dissertação (Mestrado) - Universidade Federal de Viçosa, Viçosa.

QUEIROZ, J. E. Parâmetros hidrodinâmicos de um solo de várzea para fins de drenagem subterrânea. 1995. 167 f. Tese (Doutorado) - Escola Superior de Agricultura Luiz de Queiroz, Piracicaba.

REICHARDT, K. Capacidade de campo. Revista Brasileira de Ciência do Solo, Campinas, v. 12, p. 211-216, 1988.

SALES, L. E. O.; FERREIRA, M. M.; OLIVEIRA, M. S.; CURI, N. Estimativa da velocidade de infiltração básica do solo. Pesquisa Agropecuária Brasileira, Brasília, v. 34, n. 11, p. 2091-2095, nov. 1999.

SAXTON, K. E.; RAWLS, W. J.; ROMBERGER, J. S.; PAPENDICK, R. I. Estimating generalized soil-water characteristics from texture. Soil Science Society of America Journal, Madison, v. 50, p. 1031-1036, 1986.

SAS INSTITUTE (Cary, Estados Unidos). Statistical Analysis System: language guide for personal computers. 6. ed. Cary, 1985. 429 p.

WALKLEY, A.; BLACK, I. A. An examination of the Degtjareff method for determining soil organic matter and a proposed modification of the chromic acid titration method. Soil Science, Baltimore, v. 37, p. 29-38, 1934.

WÖSTEN, J. H. M.; SCHUREN, C. H. J. E.; BOUMA, J.; STEIN, A. Functional sensitivity analysis of four methods to generate soil hydraulic functions. Soil Science Society of America Journal, Amsterdam, v. 55, p. 832836,1990 .

ZHUANG, J.; JIN, Y.; MIYAZAKI, T. Estimating water retention characteristic from soil particle-size distribution using a non-similar media concept. Soil Science, Baltimore, v. 166, n. 5, p. 308-321, 2001. 